\title{
Ethnologies
}

Seul et avec l'autre. La vie en colocation dans un quartier populaire de Québec. Par Madeleine Pastinelli. (Québec, CELAT et Presses de l'Université Laval, collection Intercultures, 2003. ISBN 2-7637-7975-1.)

\section{Marie-Blanche Fourcade}

Volume 25, numéro 2, 2003

Language and Culture / Langue et culture

URI : https://id.erudit.org/iderudit/008069ar

DOI : https://doi.org/10.7202/008069ar

Aller au sommaire du numéro

Éditeur(s)

Association Canadienne d'Ethnologie et de Folklore

ISSN

1481-5974 (imprimé)

1708-0401 (numérique)

Découvrir la revue

Citer ce compte rendu

Fourcade, M.-B. (2003). Compte rendu de [Seul et avec l'autre. La vie en colocation dans un quartier populaire de Québec. Par Madeleine Pastinelli. (Québec, CELAT et Presses de l'Université Laval, collection Intercultures, 2003. ISBN 2-7637-7975-1.)]. Ethnologies, 25(2), 278-281.

https://doi.org/10.7202/008069ar d'utilisation que vous pouvez consulter en ligne. 
connaître la sensibilité, l'imaginaire et les structures mentales des Français de l'époque.

Chaque chapitre de l'ouvrage est construit avec sa propre démarche interne. Le lecteur qui n'a pas une connaissance quelque peu étoffée de l'histoire sociale, économique et politique de l'époque pourra parfois se sentir oublié par l'auteur. En ce sens, certaines références à des ouvrages de base seraient nécessaires. De même, plusieurs des raisonnements de l'auteur seront plus parlants au lecteur qui possède les principes de base de la rhétorique. Mais cette étude demeure sans aucun doute très utile à celui qui travaille sur les débuts de la NouvelleFrance et qui veut bien comprendre la façon dont les grands voyageurs s'exprimaient par écrit. Il s'agit là d'une très bonne introduction aux sources du début du XVII ${ }^{\mathrm{e}}$ siècle.

Jean-François Plante

Université Laval

Québec

Seul et avec l'autre. La vie en colocation dans un quartier populaire de Québec. Par Madeleine Pastinelli. (Québec, CELAT et Presses de I'Université Laval, collection Intercultures, 2003. ISBN 2-7637-7975-1.)

La rentrée scolaire 2003 a vu fleurir parmi ses nouveautés un petit guide qui mérite attention. Intitulé «Contrat d'entente entre colocataires, prévenir et régler les conflits », le volume propose les bases d'un contrat entre nouveaux colocataires afin de débuter sereinement une vie commune. Au-delà des aspects concrets, l'ouvrage fait surtout prendre conscience que le principe de la cohabitation «non familiale » s'est considérablement développé ces dernières années au Québec, engendrant au sein de chaque habitation partagée de nouveaux rapports et des pratiques quotidiennes diversifiées. Il faut voir dans ce mode d'habiter devenu si populaire, en particulier auprès des étudiants et des jeunes travailleurs, un phénomène social révélateur de son temps qu'il est désormais essentiel d'analyser afin de comprendre les nouvelles sensibilités de notre société.

C'est ce que Madeleine Pastinelli, doctorante en ethnologie de l'Université Laval, travaillant sur les pratiques de sociabilité électronique, s'était lancé comme défi dans le cadre de sa recherche de 
maîtrise effectuée dans le quartier de Limoilou à Québec et publiée au printemps dernier dans la collection Intercultures des Presses de l'Université Laval. Elle nous propose de suivre le parcours de douze colocataires afin d'en faire l'expérience à travers leurs recherches de logement, l'organisation quotidienne, l'aménagement de l'espace et, plus individuellement, les perceptions qu'ils peuvent avoir de l'avenir. La réflexion part ainsi du phénomène de groupe, du mode de vie, pour progressivement s'intéresser à la trajectoire de chacun.

Le lecteur est tout d'abord convié dans les coulisses de la recherche en explorant les fondements de l'étude et de la méthode d'enquête. Comme l'explique Madeleine Pastinelli, l'entretien compréhensif pratiqué durant l'enquête était un moyen idéal de comprendre la situation, le plus justement possible, de l'intérieur. Puis, par la mise au point des concepts utilisés, comme le ménage, le couple ou la famille, on réalise rapidement que la colocation n'est pas si étrangère à nos habitudes et qu'elle emprunte allégrement aux formes plus traditionnelles des structures sociales. Il y a donc quelques traces de la famille, du célibat ou du couple dans la colocation, reste à savoir en quelles proportions et selon quelles configurations. Tout en cherchant à savoir si « les ménages non familiaux représentent un substitut de la conjugalité, une façon d'échapper aux modèles de référence ou au contraire, s'il s'agit d'une forme temporaire de compensation dans l'attente d'une nouvelle situation ou, encore, d'un nouveau modèle qui dispose de sa logique propre » (34), l'auteure analyse l'ensemble des facteurs qui pourraient façonner un modèle plus général de ce mode d'habitation. Le premier chapitre, à la fois théorique et pratique, élabore ainsi un double discours qui s'adresse tant à des chercheurs intéressés par de nouveaux terrains qu'à un public curieux d'en savoir davantage sur un phénomène vécu si naturellement et sur lequel on ne semble pas se poser de questions.

Le second chapitre est consacré à une description ethnographique de la colocation. Un état des lieux de ce «style de vie » est d'abord constitué. Le portrait socio-économique des informateurs laisse penser, au vu de la diversité des profils, que toute personne est un colocataire potentiel. Un point commun réunit pourtant l'ensemble des personnes interrogées : l'instabilité, qu'elle soit affective, financière, sociale ou professionnelle. L'habitation partagée apparaît alors comme une réponse ingénieuse et temporaire à une circonstance donnée. Le fort attachement des informateurs à leur quartier, Limoilou, est évoqué à travers l'un des 
sous-titres du chapitre : «Le village en ville» (65). En effet, l'idée de village dans lequel les individus se connaissent et se fréquentent, chassant ainsi l'éventuelle solitude, se retrouve dans la colocation qui entraîne un partage communautaire de l'habitat et un dense réseau de sociabilité. L'importance de l'espace est clairement évoquée dans le contexte de changement perpétuel : "Les occupants défilent, mais le lieu demeure le même pour ceux qui restent et pour les autres", lorsque l'appartement devient alors le territoire signifiant d'un groupe.

Le chapitre suivant resserre son champ d'exploration pour se concentrer sur les rapports des informateurs avec l'espace à partager et le colocataire. La quotidienneté de la colocation est analysée par les pratiques qui entourent l'appropriation de l'espace domestique, que ce soit par la recherche du logement, l'acquisition du mobilier et la création d'un décor de vie. On comprend que l'investissement des informateurs dans l'intérieur domestique est dépendant du sentiment de «chez-soi » qui est lui-même influencé par des facteurs tels que la durée de résidence et de sa projection dans le futur. Rester un an ou plus encourage alors la réfection des peintures, la mise en place d'une décoration permanente, voire l'achat de meubles. Après avoir passé en revue les paramètres de l'organisation d'un appartement, que ce soit le ménage ou encore la nourriture, la dernière partie du chapitre s'est principalement intéressée aux rapports entre colocataires qui nécessitent ententes, négociations et respect. L'un des points marquants de ces relations est le développement d'une "proximité distante "(127) qui implique une certaine intimité, une entente privilégiée, mais de circonstance, qui pourra disparaître à la fin du bail. Outre les choses du quotidien, les colocataires n'ont aucun autre engagement entre eux. Le titre du livre, Seul et avec l'autre, s'impose alors. Le « seul » réfère à cette absence d'autres engagements que celui de vivre et suivre les règles communes définies avec "l'autre ». L'analyse se recentre dès lors sur l'individu, annonçant un intérêt particulier pour les projets personnels que chacun formule pour l'avenir.

Le dernier chapitre fait office de bilan, telle une année de colocation terminée. Il est alors possible de voir comment les personnes se perçoivent et se projettent dans l'avenir. Le point de vue a été modifié et l'on ne cherche plus à considérer l'individu dans la colocation mais plutôt la colocation dans le parcours de l'individu, c'est-à-dire un palliatif à la solitude et un remède contre la peur de l'avenir. Cet avenir incertain est plus particulièrement lié à une situation affective : être libre ou 
engagé, seul ou en couple, avec ou sans enfant? La plupart des informateurs oscille entre toutes ces possibilités et tente de les évacuer en évitant, d'une part, de faire des projets, ce que Madeleine Pastinelli formule comme «impenser l'avenir » (138) et, d'autre part, rêve à une vie meilleure qui comporte certains clichés du bien-être et de la sérénité, tels que la vie à la campagne, une maison et des enfants. L'avenir n'appartient pas à la colocation qui est, aux yeux des informateurs, « une parenthèse à refermer» (145). La vie partagée est ainsi ressentie comme une période de transition durant laquelle il n'y a ni engagement, ni construction, mais tout simplement une vie en suspens. Des questions fondamentales sont abordées sur la trajectoire de vie. On ne parle pas seulement d'habitation mais de couple, de célibat et de nouveaux moyens de vivre ensemble. Le chapitre nous permet alors de concevoir différentes avenues de la relation à l'autre.

Loin d'être considérée comme une pratique mystérieuse, la colocation méritait d'être davantage connue et comprise au-delà des considérations pratiques et c'est ce qui a été habilement fait par Madeleine Pastinelli. Plus qu'une question de vie commune, il s'agissait là de réfléchir différemment à la relation entre le Soi et l'Autre. L'Autre si généralement vu comme l'étranger à sa culture alors qu'il peut aussi s'incarner dans le colocataire qui partage notre intimité. 